PROCEEDINGS OF THE

AMERICAN MATHEMATICAL SOCIETY

Volume 135, Number 8, August 2007, Pages 2591-2597

S 0002-9939(07)08854-5

Article electronically published on February 9, 2007

\title{
STABILITY PROBLEM FOR NUMBER-THEORETICALLY MULTIPLICATIVE FUNCTIONS
}

\author{
TOMASZ KOCHANEK AND MICHAє LEWICKI
}

(Communicated by Jonathan M. Borwein)

\begin{abstract}
We deal with the stability question for multiplicative mappings in the sense of number theory. It turns out that the conditional stability assumption:$$
|f(x y)-f(x) f(y)| \leq \varepsilon \quad \text { for relatively prime } x, y
$$

implies that $f$ lies near to some number-theoretically multiplicative function. The domain of $f$ can be general enough to admit, in special cases, the reduction of our result to the well known J. A. Baker - J. Lawrence - F. Zorzitto superstability theorem.
\end{abstract}

\section{INTRODUCTION}

In number theory a very important role is played by multiplicative functions, which in that theory are defined as follows.

Definition 1. A function $f: \mathbb{N} \rightarrow \mathbb{C}$ is called number-theoretically multiplicative (briefly: nt-multiplicative), if and only if $f \neq 0$ and

$$
f(x y)=f(x) f(y)
$$

for every $x, y \in \mathbb{N}$ such that $(x, y)=1$ (here the symbol $(x, y)$ stands for the greatest common divisor of $x$ and $y$ ).

The stability question we are concerned with reads as follows: Does there exist a function $\varphi: \mathbb{R}_{+} \rightarrow \mathbb{R}_{+}$such that $\lim _{\varepsilon \rightarrow 0_{+}} \varphi(\varepsilon)=0$ and having the property: for every function $f: \mathbb{N} \rightarrow \mathbb{C}$, satisfying

$$
((x, y)=1) \Rightarrow(|f(x y)-f(x) f(y)| \leq \varepsilon)
$$

for all $x, y \in \mathbb{N}$ and with some fixed positive constant $\varepsilon$, there exists an ntmultiplicative function $\widetilde{f}$ such that $\|f-\widetilde{f}\|_{\text {sup }} \leq \varphi(\varepsilon)$ ? It is clear that every bounded function $f: \mathbb{N} \rightarrow \mathbb{C}$ satisfies (2) with some $\varepsilon>0$, so we may leave that uninteresting case out, assuming that $f$ is an unbounded function.

We are going to prove that the answer for such a question is satisfactory. The desired function is $\varphi(\varepsilon) \equiv \varepsilon$ and, furthermore, under some additional assumptions on the function $f$ (concerning its unboundedness) we get the so called superstability effect: $f$ is necessarily nt-multiplicative.

By $P$ we denote the set of all prime numbers.

Received by the editors May 1, 2006.

2000 Mathematics Subject Classification. Primary 39B82.

Key words and phrases. Conditional functional equation, stability, arithmetic functions.

(C)2007 American Mathematical Society 2591

Reverts to public domain 28 years from publication 
Definition 2. For an arbitrary $p \in P$ we define a function $\operatorname{ord}_{p}: \mathbb{N} \rightarrow \mathbb{N} \cup\{0\}$ as

$$
\operatorname{ord}_{p}(n):=\max \left\{k \in \mathbb{N} \cup\{0\}: p^{k} \mid n\right\}, \quad n \in \mathbb{N} .
$$

\section{MAIN RESUlT}

Let us start with an auxiliary lemma.

Lemma. Assume that an unbounded function $f: \mathbb{N} \rightarrow \mathbb{C}$ satisfies (2). Then at least one of the following conditions holds:

(a) for every $x \in \mathbb{N}$ there is a sequence $\left(x_{m}\right)_{m=1}^{\infty} \in \mathbb{N}^{\mathbb{N}}$ such that $\left(x, x_{m}\right)=1$ for $m=1,2, \ldots$ and $\lim _{m \rightarrow \infty}\left|f\left(x_{m}\right)\right|=\infty$;

(b) $\quad R:=\left\{p \in P:\left.f\right|_{\left\{p^{s}: s \in \mathbb{N}\right\}}\right.$ is unbounded $\} \neq \emptyset$.

Proof. Assume that case (b) does not occur. Hence, for every $p \in P$ there exists a constant $M_{p} \in \mathbb{R}_{+}$such that $\left|f\left(p^{s}\right)\right|<M_{p}$ for $s \in \mathbb{N}$. Fix arbitrarily an $x \in \mathbb{N}$. Let $x=p_{1}^{\alpha_{1}} \cdot \ldots \cdot p_{k}^{\alpha_{k}}$ be its canonical factorization, i.e. $k \in \mathbb{N} \cup\{0\}, p_{1}, \ldots, p_{k}$ are pairwise different primes and $\alpha_{1}, \ldots, \alpha_{k} \in \mathbb{N}$. Since $f$ is unbounded, we can choose a sequence $\left(z_{m}\right)_{m=1}^{\infty} \in \mathbb{N}^{\mathbb{N}}$ such that $\lim _{m \rightarrow \infty}\left|f\left(z_{m}\right)\right|=\infty$. If $k=0$, then $x=1$ and the sequence $\left(z_{m}\right)_{m=1}^{\infty}$ fulfils case (a). So, assume that $k \geq 1$. Define a sequence $\left(\widetilde{z}_{m}\right)_{m=1}^{\infty} \in \mathbb{N}^{\mathbb{N}}$ as follows:

$$
\widetilde{z}_{m}:=\frac{z_{m}}{p_{1}^{\operatorname{ord}_{p_{1}}\left(z_{m}\right)}}, \quad m \in \mathbb{N} .
$$

Since $\left(\widetilde{z}_{m}, p_{1}\right)=1$ for $m \in \mathbb{N}$, we have

$$
\begin{aligned}
\left|f\left(z_{m}\right)\right| & =\left|f\left(p_{1}^{\operatorname{ord}_{p_{1}}\left(z_{m}\right)} \widetilde{z}_{m}\right)\right| \\
& =\left|f\left(p_{1}^{\operatorname{ord}_{p_{1}}\left(z_{m}\right)} \widetilde{z}_{m}\right)-f\left(p_{1}^{\operatorname{ord}_{p_{1}}\left(z_{m}\right)}\right) f\left(\widetilde{z}_{m}\right)+f\left(p_{1}^{\operatorname{ord}_{p_{1}}\left(z_{m}\right)}\right) f\left(\widetilde{z}_{m}\right)\right| \\
& \leq\left|f\left(p_{1}^{\operatorname{ord}_{p_{1}}\left(z_{m}\right)} \widetilde{z}_{m}\right)-f\left(p_{1}^{\operatorname{ord}_{p_{1}}\left(z_{m}\right)}\right) f\left(\widetilde{z}_{m}\right)\right|+\left|f\left(p_{1}^{\operatorname{ord}_{p_{1}}\left(z_{m}\right)}\right)\right|\left|f\left(\widetilde{z}_{m}\right)\right| \\
& \leq \varepsilon+M_{p_{1}}\left|f\left(\widetilde{z}_{m}\right)\right| .
\end{aligned}
$$

Consequently,

$$
\left|f\left(\widetilde{z}_{m}\right)\right| \geq \frac{\left|f\left(z_{m}\right)\right|-\varepsilon}{M_{p_{1}}}
$$

and the right-hand side tends to infinity as $m \rightarrow \infty$.

Thus, we have constructed a sequence $\left(\widetilde{z}_{m}\right)_{m=1}^{\infty}$ such that

(i) $\lim _{m \rightarrow \infty}\left|f\left(\widetilde{z}_{m}\right)\right|=\infty$;

(ii) all prime factors of $\widetilde{z}_{m}$ are also prime factors of $z_{m}$, for all $m \in \mathbb{N}$;

(iii) none of elements of $\left(\widetilde{z}_{m}\right)_{m=1}^{\infty}$ is divisible by $p_{1}$.

Repeating that construction $k$ times we will get a sequence $\left(x_{m}\right)_{m=1}^{\infty}$ such that case (a) of the assertion is satisfied. Since $x \in \mathbb{N}$ is arbitrary, it proves that negation of case (b) implies case (a). This ends the proof.

Observe that under the above assumptions, if case (a) fails to hold, then $R$ is nonempty and finite. Indeed, the Lemma implies that $R \neq \emptyset$, and if $R$ were infinite, then for every $x \in \mathbb{N}$ we could choose a sequence satisfying case (a), since $x$ has only a finite number of primes in its canonical factorization. For the sake of brevity, if in the sequel $x \in \mathbb{N},\left(x_{m}\right)_{m=1}^{\infty} \in \mathbb{N}^{\mathbb{N}}, \lim _{m \rightarrow \infty}\left|f\left(x_{m}\right)\right|=\infty$ and $\left(x, x_{m}\right)=1$ for $m \in \mathbb{N}$, we simply say that $\left(x_{m}\right)_{m=1}^{\infty}$ satisfies (a) with $x$. 
Theorem. Assume that an unbounded function $f: \mathbb{N} \rightarrow \mathbb{C}$ satisfies (2). Then there exists an nt-multiplicative function $\widetilde{f}$ such that

$$
|f(x)-\widetilde{f}(x)| \leq \varepsilon, \quad x \in \mathbb{N} .
$$

Moreover, in the case where condition (a) of the Lemma holds true, then

$$
f=\widetilde{f} \text {. }
$$

Generally, we have

$$
\left.f\right|_{\bigcup_{p \in R} \operatorname{ord}_{p}^{-1}(0)}=\left.\widetilde{f}\right|_{\bigcup_{p \in R} \operatorname{ord}_{p}^{-1}(0)} .
$$

Proof. First, assume that case (a) is satisfied. We have to show that $f$ is ntmultiplicative (that is, nothing else but (4)). Fix arbitrarily an $x \in \mathbb{N}$. Since (a) holds, we can choose a sequence $\left(x_{m}\right)_{m=1}^{\infty} \in \mathbb{N}^{\mathbb{N}}$ such that $\left(x, x_{m}\right)=1$ for $m \in \mathbb{N}$ and $\lim _{m \rightarrow \infty}\left|f\left(x_{m}\right)\right|=\infty$. By inequality (2), we have

$$
\left|f\left(x_{m} x\right)-f\left(x_{m}\right) f(x)\right| \leq \varepsilon
$$

for all $m \in \mathbb{N}$. For sufficiently large $m$ we have $\left|f\left(x_{m}\right)\right| \neq 0$. For those $m$ we divide both sides by $\left|f\left(x_{m}\right)\right|$. This yields

$$
\left|\frac{f\left(x_{m} x\right)}{f\left(x_{m}\right)}-f(x)\right| \leq \frac{\varepsilon}{\left|f\left(x_{m}\right)\right|}
$$

for sufficiently large $m \in \mathbb{N}$. Hence

$$
\lim _{m \rightarrow \infty} \frac{f\left(x_{m} x\right)}{f\left(x_{m}\right)}=f(x),
$$

which is true for every $x \in \mathbb{N}$ and every sequence $\left(x_{m}\right)_{m=1}^{\infty}$ satisfying (a) with $x$. In the sequel $r(t, u)$ denotes the difference $f(t u)-f(t) f(u)$ for $t, u \in \mathbb{N}$. Fix $x, y \in \mathbb{N}$ such that $(x, y)=1$. Let $\left(a_{m}\right)_{m=1}^{\infty}$ be a sequence satisfying (a) with $x y$. Since $\left(x y, a_{m}\right)=1$ implies both $\left(x, a_{m}\right)=1$ and $\left(y, a_{m}\right)=1$ for all $m \in \mathbb{N}$, we infer that the sequence $\left(a_{m}\right)_{m=1}^{\infty}$ satisfies (a) with $x$ and $y$ as well. By (6) we have

$$
\begin{aligned}
f(x y) & =\lim _{m \rightarrow \infty} \frac{f\left(a_{m} x y\right)}{f\left(a_{m}\right)} \\
& =\lim _{m \rightarrow \infty} \frac{1}{f\left(a_{m}\right)}\left(f\left(a_{m} x\right) f(y)+r\left(a_{m} x, y\right)\right) .
\end{aligned}
$$

Since $\left(a_{m}, y\right)=(x, y)=1$ implies $\left(a_{m} x, y\right)=1$, assumption (2) yields $\left|r\left(a_{m} x, y\right)\right| \leq$ $\varepsilon$. So,

$$
\lim _{m \rightarrow \infty} \frac{r\left(a_{m} x, y\right)}{f\left(a_{m}\right)}=0 .
$$

Thus and by (6)

$$
f(x y)=f(y) \lim _{m \rightarrow \infty} \frac{f\left(a_{m} x\right)}{f\left(a_{m}\right)}=f(x) f(y),
$$

which states that $f$ is nt-multiplicative.

Now assume that case (a) fails to hold. Then, by the Lemma, the set $R$ is nonempty and, as has already been remarked, $R$ is finite. Thus the definition

$$
I:=\prod_{p \in R} p
$$


is correct. Define

$$
S:=\bigcup_{p \in R} \operatorname{ord}_{p}^{-1}(0)
$$

( $S$ is simply the set of all those elements of $\mathbb{N}$ that are not divisible by at least one prime from $R$ ). Observe that $\mathbb{N} \backslash S=\bigcap_{p \in R}\{p x: x \in \mathbb{N}\}$, hence $\mathbb{N}=S \cup(I)$, where (I) denotes the ideal generated by $I$.

For $s \in S$ we put $\widetilde{f}(s):=f(s)$. We shall show that $f$ is nt-multiplicative in $S$ in the following sense: if $x, y, x y \in S$ and $(x, y)=1$, then $f(x y)=f(x) f(y)$. Observe that for every $s \in S$ we can choose a prime $p_{s} \in R$ such that $\left(s, p_{s}\right)=1$; hence $\left(s, p_{s}^{m}\right)=1$ for all $m \in \mathbb{N}$. Since $f$ restricted to the set $\left\{p_{s}^{m}: m \in \mathbb{N}\right\}$ is unbounded, there exists a subsequence $k_{1}<k_{2}<\ldots$ of $\mathbb{N}$ such that

$$
\lim _{m \rightarrow \infty}\left|f\left(p_{s}^{k_{m}}\right)\right|=\infty \text {. }
$$

By (2), we have

$$
\left|f\left(p_{s}^{k_{m}} s\right)-f\left(p_{s}^{k_{m}}\right) f(s)\right| \leq \varepsilon
$$

for all $m \in \mathbb{N}$, whereas by $(7), f\left(p_{s}^{k_{m}}\right) \neq 0$ for sufficiently large $m \in \mathbb{N}$. Dividing both sides of the previous inequality by $\left|f\left(p_{s}^{k_{m}}\right)\right|$ for those $m \in \mathbb{N}$ and making use of $(7)$, we obtain

$$
\lim _{m \rightarrow \infty} \frac{f\left(p_{s}^{k_{m}} s\right)}{f\left(p_{s}^{k_{m}}\right)}=f(s),
$$

which is true for all $s \in S$ and all sequences $\left(p_{s}^{k_{m}}\right)_{m=1}^{\infty}$ satisfying (7) and such that $\left(s, p_{s}\right)=1$. Now, fix $x, y \in S$ such that $x y \in S$ and $(x, y)=1$. The reasoning leading to equation (8) shows that for a prime $p_{x y}$ which divides neither $x$ nor $y$ and for a subsequence $k_{1}<k_{2}<\ldots$ of $\mathbb{N}$ we have $\lim _{m \rightarrow \infty}\left|f\left(p_{x y}^{k_{m}}\right)\right|=\infty$ and $f(x y)=\lim _{m \rightarrow \infty} \frac{f\left(p_{x y}^{k_{m}} x y\right)}{f\left(p_{x y}^{k_{m}}\right)}$. Since $\left(p_{x y}, y\right)=(x, y)=1$ implies $\left(p_{x y} x, y\right)=1$, assumption (2) yields $\left|r\left(p_{x y} x, y\right)\right| \leq \varepsilon$. So, $\lim _{m \rightarrow \infty} \frac{r\left(p_{x y}^{k_{m}} x, y\right)}{f\left(p_{x y}^{k m}\right)}=0$ and

$$
\begin{aligned}
f(x y) & =\lim _{m \rightarrow \infty} \frac{f\left(p_{x y}^{k_{m}} x y\right)}{f\left(p_{x y}^{k_{m}}\right)}=\lim _{m \rightarrow \infty} \frac{1}{f\left(p_{x y}^{k_{m}}\right)}\left[f\left(p_{x y}^{k_{m}} x\right) f(y)+r\left(p_{x y}^{k_{m}} x, y\right)\right] \\
& =f(y) \lim _{m \rightarrow \infty} \frac{f\left(p_{x y}^{k_{m}} x\right)}{f\left(p_{x y}^{k_{m}}\right)}=f(x) f(y) .
\end{aligned}
$$

We have just proved that $f$ is nt-multiplicative in $S$.

Let $I=p_{1} \cdot \ldots \cdot p_{k}$ be the canonical factorization of $I$ (i.e. $R=\left\{p_{1}, \ldots, p_{k}\right\}, 0<$ $k<\infty)$. If $k>1$, then we have already defined all of the values: $\widetilde{f}\left(p_{1}^{\alpha_{1}}\right), \ldots, \widetilde{f}\left(p_{k}^{\alpha_{k}}\right)$ for every $\alpha_{1}, \ldots, \alpha_{k} \in \mathbb{N}$, since all previous arguments belong to $S$. We have also defined the value $\widetilde{f}(1)=1$ (note that (2) and unboundedness of $f$ easily imply that $f(1)=1$ ). Making $\widetilde{f}$ to be nt-multiplicative, we have to define

$$
\begin{aligned}
\widetilde{f}\left(q_{1}^{\kappa_{1}} \cdot \ldots \cdot q_{l}^{\kappa_{l}}\right): & =\widetilde{f}\left(q_{1}^{\kappa_{1}}\right) \cdot \ldots \cdot \widetilde{f}\left(q_{l}^{\kappa_{l}}\right) \\
& =f\left(q_{1}^{\kappa_{1}}\right) \cdot \ldots \cdot f\left(q_{l}^{\kappa_{l}}\right)
\end{aligned}
$$

for all pairwise different primes $q_{1}, \ldots, q_{l}$ and naturals $\kappa_{1}, \ldots, \kappa_{l}$. For $k=1$ we define $\widetilde{f}\left(p_{1}^{\kappa}\right):=f\left(p_{1}^{\kappa}\right)(\kappa \in \mathbb{N})$, and having already defined values of $\widetilde{f}$ on all powers of primes we similarly put other values like in (9). We have considered all possible values of $k$, because - by the Lemma - we have $k>0$. The fact that the above 
definition is correct (i.e. it is compatible with (5)) follows from nt-multiplicativity of $f$ in $S$.

It remains to show inequality (3). Fix $x \in \mathbb{N}$. It has a representation of the form

$$
x=q_{1}^{\alpha_{1}} \cdot \ldots \cdot q_{l}^{\alpha_{l}}
$$

where $q_{1}, \ldots, q_{l}$ are pairwise different primes and $\alpha_{1}, \ldots, \alpha_{l} \in \mathbb{N}$. Since the functions $f$ and $\widetilde{f}$ coincide on prime powers and $\widetilde{f}$ is nt-multiplicative, we have

$$
|f(x)-\tilde{f}(x)|=\left|f\left(q_{1}^{\alpha_{1}} \cdot \ldots \cdot q_{l}^{\alpha_{l}}\right)-f\left(q_{1}^{\alpha_{1}}\right) \cdot \ldots \cdot f\left(q_{l}^{\alpha_{l}}\right)\right| .
$$

If none of $q_{1}, \ldots, q_{l}$ belongs to $R$, then $x \in S$ and the left-hand side of (10) is equal to 0 , by nt-multiplicativity of $f$ in $S$. If $q_{i} \in R$ for some $i \in\{1, \ldots, l\}$, then

$$
\prod_{j=1, j \neq i}^{l} q_{j}^{\alpha_{j}} \in S
$$

whence nt-multiplicativity of $f$ in $S$ implies that

$$
f\left(\prod_{j=1, j \neq i}^{l} q_{j}^{\alpha_{j}}\right)=\prod_{j=1, j \neq i}^{l} f\left(q_{j}^{\alpha_{j}}\right) .
$$

Plainly,

$$
\left(\prod_{j=1, j \neq i}^{l} q_{j}^{\alpha_{j}}, q_{i}^{\alpha_{i}}\right)=1
$$

so, by virtue of (2), we have

$$
\left|f\left(\prod_{j=1, j \neq i}^{l} q_{j}^{\alpha_{j}} \cdot q_{i}^{\alpha_{i}}\right)-f\left(\prod_{j=1, j \neq i}^{l} q_{j}^{\alpha_{j}}\right) f\left(q_{i}^{\alpha_{i}}\right)\right| \leq \varepsilon .
$$

Comparing it to (11) and (10) we get (3), which ends the proof.

\section{REMARKS AND POSSIBLE GENERALIZATION}

Remark 1. It is easily seen that in the case when $f$ is a real function, the function $\widetilde{f}$ constructed in the proof of the Theorem is real as well.

Remark 2 . We may replace the standard domain $\mathbb{N}$ of equation (1) by an arbitrary semigroup with unique factorization which we define as follows.

Let $(P,+, \cdot, 0,1)$ be an integral domain with unique factorization and let $(D, \cdot)$ be a subsemigroup of the semigroup $(P \backslash\{0\}, \cdot)$ which contains the identity. If $x, y \in D$, we say that $x$ divides $y$ (and write $x \mid y$ ) iff $y=a x$ for some $a \in D$. We say that $x$ is equivalent to $y$ iff $x \mid y$ and $y \mid x$. In such a case $y=a x, x=b y$ for some $a, b \in D$, whence $y=a b y$ and $a b=1, b=a^{-1}$. Thus $a$ is an invertible element which belongs to $D$ together with its inverse. A noninvertible element $z \in D$ is called unfactorable iff it cannot be represented as a product of two noninvertible elements of $D$; it is called prime iff the equality $z=a_{1} a_{2}$ for some $a_{1}, a_{2} \in D$ implies $z \mid a_{1}$ or $z \mid a_{2}$. It is easily proved that these two notions are equivalent in semigroups with unique factorization as well as in integral domains. 
Definition 3. A subsemigroup $(D, \cdot)$ of the semigroup $(P \backslash\{0\}, \cdot)$ is called a semigroup with unique factorization, if and only if each of its noninvertible element has exactly one representation (up to a permutation and equivalence of factors) as a product of prime elements of that subsemigroup (equivalently: elements unfactorable in $D$ ).

In semigroups with unique factorization the notion of greatest common divisor can be introduced as it is done for integral domains with unique factorization. The greatest common divisor is determined up to the equivalence relation, e.g. $(x, y)=1$ means exactly that there does not exist a prime element dividing both $x$ and $y$. A suitable modification of Definition 1 may be considered for nt-multiplicative functions with a domain $D$ being a semigroup with unique factorization. A condition for functions $f: D \rightarrow \mathbb{C}$ which is analogous to (2) may be considered as well. One may easily verify that the above argumentations work in such a more general case without any significant modifications. We only have to work with a set $P(D)$ - any maximal subsets of $D$ containing pairwise nonequivalent prime elements, instead of the set $P$, and invertible elements of $D$, different from 1 , have to be taken into account in canonical factorizations.

Such a generalization may be motivated by the fact, that - although $(\mathbb{N}, \cdot)$ is the most important and natural semigroup with unique factorization - there exist many other such semigroups (like $(\mathbb{Z}[i], \cdot)$ or $\left(K\left[X_{1}, \ldots, X_{n}\right], \cdot\right)$ for instance). Every field is also an example of a semigroup with unique factorization, because the requirement of factorization uniqueness is trivially fulfilled, since all of its nonzero elements are invertible. Our result (which concerns conditional functional equations) applied for fields became a result concerning classical stability (unconditional).

Remark 3. In view of assertion (4), the Theorem establishes something more than the stability of the equation of nt-multiplicative functions. More precisely, the sufficient condition for getting effect (4) is just the unboundedness of $f$ restricted to some subset $A \subseteq \mathbb{N}$ consisting of infinitely many mutually relatively prime elements.

It is known that approximately multiplicative functions $f: S \rightarrow \mathbb{C}$ have the property that they are either bounded or multiplicative, for an arbitrary semigroup $(S, \cdot)$ (see Theorem 1 in the paper of J. A. Baker, J. Lawrence and F. Zorzitto 1 ] and Theorem 1 in the paper of J. A. Baker [2] which strengthens the previous one). Observe that if $(K,+, \cdot, 0,1)$ is a field, then $(K \backslash\{0\}, \cdot)$ is a semigroup with unique factorization where the condition $(x, y)=1$ is trivially fulfilled for all $x, y \in K \backslash\{0\}$. In such a situation our Theorem reduces to some special cases of the just mentioned J. A. Baker - J. Lawrence - F. Zorzitto theorem.

Example 1. As in the paper of J. A. Baker, the range of $f$ cannot be replaced by an arbitrary normed algebra. To show this, we apply a slight modification of the counterexample given in [2]. Let $A:=\left\{\left[\begin{array}{ll}a & b \\ c & d\end{array}\right]: a, b, c, d \in \mathbb{R}\right\}$ with a norm $\|\cdot\|: A^{2} \rightarrow[0, \infty)$ given by $\left\|\left[\begin{array}{ll}a & b \\ c & d\end{array}\right]\right\|:=\sqrt{a^{2}+b^{2}+c^{2}+d^{2}}$. Let $\phi: \mathbb{N} \rightarrow \mathbb{N}$ be Euler's function and let $\eta \in \mathbb{R}$ satisfy $\eta-\eta^{2}=\varepsilon$ where $\varepsilon>0$ is fixed. Define $f: \mathbb{N} \rightarrow A$ as

$$
f(n):=\left[\begin{array}{cc}
\phi(n) & 0 \\
0 & \eta
\end{array}\right], \quad n \in \mathbb{N} .
$$


The function $f$ satisfies (2) with $R$ equal to the set of all prime numbers, but contrary to (4) $f$ is not nt-multiplicative, since

$$
\|f(m n)-f(m) f(n)\|=\left\|\left[\begin{array}{cc}
0 & 0 \\
0 & \eta-\eta^{2}
\end{array}\right]\right\|=\varepsilon
$$

for $m, n \in \mathbb{N}$ such that $(m, n)=1$.

Example 2. Finally we show that the constant $\varepsilon$ in (3) is sharp. To this end define a function $f: \mathbb{N} \rightarrow \mathbb{R}$ as follows. Fix arbitrarily two different primes $p$ and $q$ and put

$$
f(n):=\left\{\begin{array}{lll}
1, & \text { if } p \nmid n, q \nmid n, & \\
\operatorname{ord}_{p}(n), & \text { if } p \mid n, q \nmid n, \\
\operatorname{ord}_{q}(n), & \text { if } p \nmid n, q \mid n, \\
\operatorname{ord}_{p}(n) \cdot \operatorname{ord}_{q}(n), & \text { if } p|n, q| n, n \neq p q, \\
2, & \text { if } n=p q,
\end{array} \quad n \in \mathbb{N} .\right.
$$

It is easy to check that $f$ fulfils (2) with $\varepsilon=1$. Let $\widetilde{f}: \mathbb{N} \rightarrow \mathbb{C}$ be any of ntmultiplicative functions satisfying $\|f-\widetilde{f}\|_{\text {sup }} \leq 1$. Let $\alpha:=\widetilde{f}(p)$. For every $k=2,3, \ldots$ we have $f\left(p q^{k}\right)=k$ hence $1 \geq\left|\widetilde{f}\left(p q^{k}\right)-k\right|=\left|\alpha \widetilde{f}\left(q^{k}\right)-k\right|$. Similarly, because of $f\left(q^{k}\right)=k$, one has $\left|\widetilde{f}\left(q^{k}\right)-k\right| \leq 1$. Let $\theta_{k}:=\widetilde{f}\left(q^{k}\right)-k$ for $k=1,2, \ldots$. The latter inequality says that the sequence $\left(\theta_{k}\right)$ is bounded: $\left|\theta_{k}\right| \leq 1, k \geq 2$, whereas the former states that

$$
\left|\alpha \theta_{k}+(\alpha-1) k\right| \leq 1, \quad k \geq 2 .
$$

The left-hand side of this inequality would tend to infinity provided that $\alpha-1$ is different from zero. We have thus shown that $\widetilde{f}(p)=1$. The symmetry of roles of $p$ and $q$ allows us to state that $\widetilde{f}(q)=1$ as well. Therefore, $\widetilde{f}(p q)=\widetilde{f}(p) \widetilde{f}(q)=1$ which implies $|f(p q)-\widetilde{f}(p q)|=1$ and hence $\|f-\widetilde{f}\|_{\text {sup }}=1$.

\section{REFERENCES}

[1] J. A. Baker, J. Lawrence, F. Zorzitto, The stability of the equation $f(x+y)=f(x) f(y)$, Proc. Amer. Math. Soc. 74 (1979), 242-246. MR0524294 (80d:39009)

[2] J. A. Baker, The stability of the cosine equation, Proc. Amer. Math. Soc. 80 (1980), 411-416. MR0580995 (81m:39015)

Institute of Mathematics, Silesian University, Bankowa 14, PL-40 007 Katowice, POLAND

E-mail address: t_kochanek@wp.pl

Institute of Mathematics, Silesian University, Bankowa 14, PL-40 007 Katowice, POLAND

E-mail address: m_lewicki@wp.pl 